\title{
Advances in uncertainty quantification for water resources applications
}

\author{
Valentina Ciriello ${ }^{1}$ (1) Jonghyun Lee ${ }^{2} \cdot$ Daniel M. Tartakovsky $^{3}$
}

Accepted: 23 February 2021 / Published online: 27 February 2021

(C) The Author(s), under exclusive licence to Springer-Verlag GmbH, DE part of Springer Nature 2021

Engineering and physical sciences often rely on mathematical approximations of reality (aka models) that are parameterized with indirect and noisy observations. Consequently, characterization and quantification of uncertainty is a primary challenge in modern science-based predictions (National Research Council 2012). When applied to water resources, uncertainty quantification (UQ) plays a central role in assessing the reliability and accuracy of hydrological models and making optimal water management decisions based on such models (Tartakovsky 2013). Environmental policy and regulations around the world increasingly require UQ as a basis for the planning of measures able to meet the prescribed objectives and minimize possible hazards for human health and the environment (e.g., EP 2000).

Recent advances in data acquisition and computational power herald a new era of breakthrough innovation but also pose several challenges. The unprecedented volumes of measurements from remote sensing and geophysics enhance our understanding of natural systems, potentially reducing uncertainty in the hydrological modeling. Yet, processing big environmental data for UQ requires significant computational effort (McCabe et al. 2017). Largescale simulation tools in high performance computing

Valentina Ciriello

v.ciriello@unibo.it

Jonghyun Lee

jonghyun.harry.lee@hawaii.edu

Daniel M. Tartakovsky

tartakovsky@stanford.edu

1 Department of Civil, Chemical, Environmental, and Materials Engineering, University of Bologna, Bologna, Italy

2 Civil and Environmental Engineering and Water Resources Research Center, University of Hawaii at Manoa, Honolulu, USA

3 Department of Energy Resources Engineering, Stanford University, Stanford, USA environments enable one to simulate complex physical and biochemical processes at multiple spatiotemporal scales (Ciriello et al. 2017, 2019), but the number of unknown parameters in such models can be on the order of several millions or billions (Hammond et al. 2014).

With these advances and challenges, new theoretical and computational approaches have been introduced to improve and guarantee the accuracy and computational scalability of UQ (Ghorbanidehno et al. 2020). In parallel with a popularity of big data analytics and open-source machine learning software packages (Zaharia et al. 2016; Abadi et al. 2016), data-driven hydrological modeling became an active area of research (Reichstein et al. 2019). It calls for theoretical investigation and algorithm development for UQ of such data-driven models.

This special issue collates the latest achievements in UQ for water resources applications. We briefly introduce the papers of this special issue below.

Althoff et al. (2021) address the topic of quantifying predictive uncertainty in hydrological models with recurrent neural networks. They propose a dropout-based approach to introduce randomness in their trained neural network (NN) model without training multiple NN models as in the typical Monte Carlo based approaches. With daily precipitation-runoff prediction applications, they compare the dropout ensemble with multiple NN model-based ensemble to illustrate the computational efficiency of the propose method while offering a reasonable accuracy.

Cheng et al. (2021) address the topic of variational data assimilation aiming to improve the estimation of background and observation error covariance matrices. Based on the advantages and limitations of several online and offline covariance matrix tuning algorithms with an convergence analysis, they propose a multi-stage tuning approach by tuning the error magnitude using the offline methods followed by correcting the covariance structure with the online approaches. Since covariance kernel and its hyperparameters are seldom available in practice, the proposed approach can be useful in engineering 
applications. With run-off forecast examples in the Tarn River, France, they show accurate and robust runoff flow prediction compared to assimilation results with single covariance tuning.

Fallico et al. (2021) study scaling behavior of commonly used aquifer parameters based on their own experimental laboratory scale data sets and existing fractal models. First, the effective hydraulic conductivity, porosity and tortuosity of their confined aquifers with different material configuration are determined from slug tests and additional data analysis. Then, fractal models are utilized to identify the scaling dependent relationships among the aquifer parameters. Through this process, the authors evaluate the accuracy and the level of uncertainty of the widely used fractal models.

Forghani et al. (2021) focus on reduced order modeling (ROM) of the shallow water equations (SWEs) combining stochastic inverse modeling and deep learning approaches. For effective ROM generation, they use posterior bathymetry samples from a Bayesian inverse modeling approach as training data sets. Then, three different approachesprincipal component analysis-based deep neural network, supervised encoder, and supervised variational encoderexploit linear and nonlinear dimension reduction-based latent space in order to construct ROM of SWEs for different bathymetry and boundary conditions. The proposed approaches are applied to flow simulation in a reach of the Savannah river, GA.

Henri et al. (2021) assess the impact of land use changes (LUCs) on the non-point source-driven groundwater quality at extraction wells using an computationally efficient approach. By assuming the LUC and spatial distribution of crops do not affect transport path ways and related travel times, they use a simple linear model to provide statistics of contaminant breakthrough curves at the wells based on the stochastic flow and transport simulation results with prior- and post-LUCs. The authors validate the accuracy of the proposed approach with full 3D stochastic simulation results of LUC scenarios and also provided conditions in which the approach works best. The proposed model is applied to test LUC scenarios for Central Valley alluvial aquifer system and was also included in an online tool for swift LUC scenario testing and related decision making.

Muniruzzaman and Pedretti (2021) review the evolution of the mechanistic modeling approaches for long-term waste rock simulations. They address different challenges related to tackling physical, chemical, and thermal heterogeneity in predictive modeling of mining systems. They point out the importance of stochastic modeling as a fundamental approach to embed heterogeneity in risk assessments and model-based decisions, and highlighted that the use of such method is no longer prohibitive because of the increasing accessibility to high performance computing resources. It is emphasized that the regulators and decision makers must be convinced of the benefit of using stochastic modeling, which is still considered to belong mainly to the academic sphere. Finally, the authors present their perspective regarding how the discipline could evolve in the next decade to increasingly include heterogeneity in acid mine drainage studies.

Morvillo et al. (2021) focus on UQ of the first arrival times of contaminants in aquifers. To reduce the computational time of stochastic flow and transport simulations, they rank randomly generated spatially heterogeneous hydraulic conductivity fields according to their connectivity. The connectivity-based ranking is formulated as the shortest path problem in a graph followed by binary search tree construction for efficient sampling of conductivity fields. Through test cases, the convergence rate of their proposed approach to the mean and standard deviation of the first arrival times was faster with a reduced computation cost than that of a Monte Carlo method.

The guest editors of this special issue thank all the authors for their contributions, anonymous reviewers for their thorough evaluations and constructive comments, the Editor-in-Chief, George Christakos, for his encouragement and support, and the editorial staff for their assistance.

\section{Declaration}

Conflict of interest The authors declare that they have no conflict of interest.

\section{References}

Abadi M, Barham P, Chen J, Chen Z, Davis A, Dean J, Devin M, Ghemawat S, Irving G, Isard M, et al. (2016) Tensorflow: A system for large-scale machine learning. In: 12th USENIX symposium on operating systems design and implementation, pp 265-283

Althoff D, Rodrigues LN, Bazame HC (2021) Uncertainty quantification for hydrological models based on neural networks: the dropout ensemble. Stoch Environ Res Risk Assess. https://doi. org/10.1007/s00477-021-01980-8

Cheng S, Argaud JP, Iooss B, Lucor D, Poncot A (2021) Error covariance tuning in variational data assimilation: application to an operating hydrological model. Stoch Environ Res Risk Assess. https://doi.org/10.1007/s00477-020-01933-7

Ciriello V, Lauriola I, Bonvicini S, Cozzani V, Di Federico V, Tartakovsky DM (2017) Impact of hydrogeological uncertainty on estimation of environmental risks posed by hydrocarbon transportation networks. Water Resour Res 53(11):8686-8697

Ciriello V, Lauriola I, Tartakovsky DM (2019) Distribution-based global sensitivity analysis in hydrology. Water Resour Res 55:8708-8720

EP, (2000) Directive 2000/60/EC of 23 October 2000 establishing a framework for Community action in the field of water policy. Tech. rep, European Parliament and Council 
Fallico C, De Bartolo S, Brunetti GFA, Severino G (2021) Use of fractal models to define the scaling behavior of the aquifers' parameters at the mesoscale. Stoch Environ Res Risk Assess. https://doi.org/10.1007/s00477-020-01881-2

Forghani M, Qian Y, Lee J, Farthing MW, Hesser T, Kitanidis P, Darve EF (2021) Application of deep learning to large scale riverine flow velocity estimation. Stoch Environ Res Risk Assess. https://doi.org/10.1007/s00477-021-01988-0

Ghorbanidehno H, Kokkinaki A, Lee J, Darve E (2020) Recent developments in fast and scalable inverse modeling and data assimilation methods in hydrology. J Hydrol 591:125266

Hammond GE, Lichtner PC, Mills RT (2014) Evaluating the performance of parallel subsurface simulators: An illustrative example with PFLOTRAN. Water Resour Res 50:208-228. https://doi.org/10.1002/2012WR013483

Henri CV, Harter T, Diamantopoulos E (2021) Stochastic assessment of the effect of land-use change on nonpoint source-driven groundwater quality using an efficient scaling approach. Stoch Environ Res Risk Assess. https://doi.org/10.1007/s00477-02001869-y

McCabe MF, Rodell M, Alsdorf DE, Miralles DG, Uijlenhoet R, Wagner W, Lucieer A, Houborg R, Verhoest NEC, Franz TE, Shi J, Gao H, Wood EF (2017) The future of earth observation in hydrology. Hydrol Earth Syst Sci 21(7):3879-3914. https://doi. org/10.5194/hess-21-3879-2017

Morvillo M, Bonazzi A, Rizzo CB, de Barros FPJ (2021) Improving the computational efficiency of first arrival time uncertainty estimation using a connectivity-based ranking Monte Carlo method. Stoch Environ Res Risk Assess. https://doi.org/10.1007/ s00477-020-01943-5

Muniruzzaman M, Pedretti D (2021) Mechanistic models supporting uncertainty quantification of water quality predictions in heterogeneous mining waste rocks: a review. Stoch Environ Res Risk Assess. https://doi.org/10.1007/s00477-020-01884-z

National Research Council (2012) Assessing the reliability of complex models: mathematical and statistical foundations of verification, validation, and uncertainty quantification. National Academies Press

Reichstein M, Camps-Valls G, Stevens B, Jung M, Denzler J, Carvalhais N et al (2019) Deep learning and process understanding for data-driven earth system science. Nature 566(7743):195-204

Tartakovsky DM (2013) Assessment and management of risk in subsurface hydrology: a review and perspective. Adv Water Resour 51:247-260. https://doi.org/10.1016/j.advwatres.2012. 04.007

Zaharia M, Xin RS, Wendell P, Das T, Armbrust M, Dave A, Meng X, Rosen J, Venkataraman S, Franklin MJ et al (2016) Apache Spark: a unified engine for big data processing. Commun ACM 59(11):56-65

Publisher's Note Springer Nature remains neutral with regard to jurisdictional claims in published maps and institutional affiliations. 